In der Rubrik „Literatur kompakt" werden die wichtigsten Originalarbeiten aus der internationalen Fachliteratur referiert.

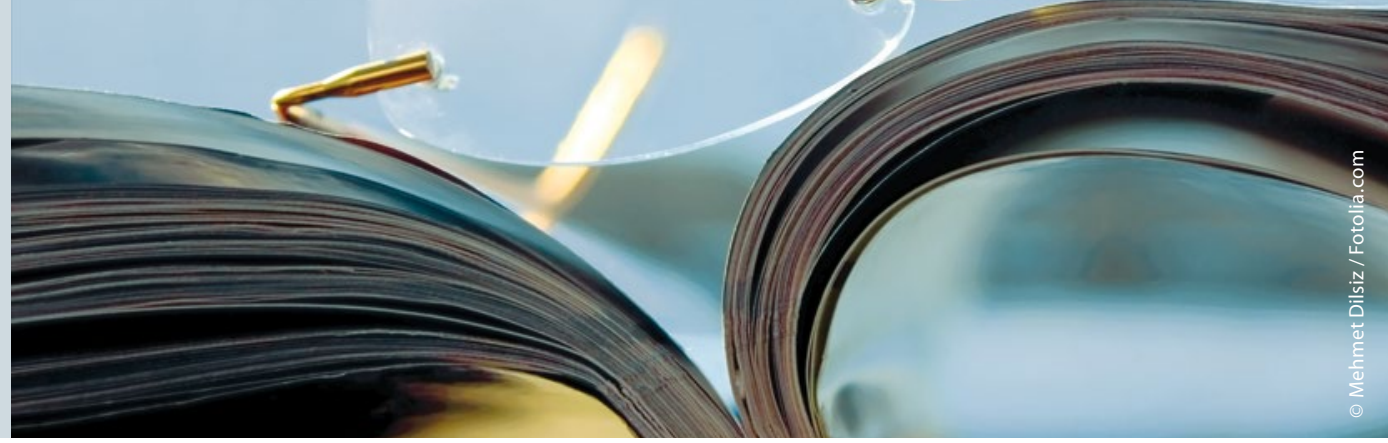

\section{Das bringt die adjuvante Chemo bei Blasenkrebs}

\author{
Mehr Patienten mit lokal fortgeschrittenem Blasenkrebs, die nach der Zystek- \\ tomie adjuvant chemotherapeutisch behandelt werden, leben noch nach \\ fünf Jahren als Patienten ohne Adjuvanz. Dieses Ergebnis einer Beobach- \\ tungsstudie bestätigt Resultate früherer Studien.
}

$\mathrm{n}$ mehreren Studien gab es Hinweise, dass Patienten mit einem lokal fortgeschrittenen Harnblasenkarzinom von einer adjuvanten Chemotherapie nach der operativen Entfernung der Harnblase profitieren. Allerdings lassen die Ergebnisse kein endgültiges Urteil zum Nutzen dieser Strategie zu. Um eine bessere Aussage als bisher treffen zu können, haben Onkologen um Dr. Matthew D. Galsky vom Tisch Cancer Institute in New York eine Beobachtungsstudie gemacht, für die sie die Daten des National Cancer Data Base (NCDB) nutzten, das in USA mit den Befunden von mehr als $70 \%$ aller neuen Krebserkrankungen gefüttert wird. Die Ärzte konzentrierten sich auf die Daten, die zwischen 2003 und 2006 gesammelt worden waren, und zwar von Patienten, bei denen pT3-T4Tumoren mit oder ohne Lymphknotenbeteiligung nach der Zystektomie diagnostiziert worden waren.

Insgesamt 5.653 Patienten aus einem Pool von fast 600.000 mit einem Harnblasenkarzinom konnten für die Studie berücksichtigt werden. $23 \%$ von ihnen, also 1.293 Patienten, erhielten nach der Operation eine adjuvante Chemotherapie. Aus den Dokumentationen geht allerdings nicht hervor, um welche Chemotherapien es sich handelte. Die NCDB enthält nur Angaben darüber, ob eine Kombinations- oder Monotherapie gemacht wurde. Median lag das Follow-up bei 6,8 (Adjuvanz) und 6,7 Jahren. Die Autoren errechneten anhand der Daten eine Fünf-Jahres-Überlebensrate von $37,0 \%$ (95\%-Konfidenzintervall zwischen 34,3\% und 39,7 \%) bei Patienten mit Adjuvanz und von 29,1 \% (95\%-Konfidenzintervall zwischen $27,7 \%$ und $30,5 \%)$ in der Vergleichsgruppe ohne Chemotherapie. Unter Anwendung des Propensity Scores, mit dem üblicherweise Faktoren, die zu statistischen Verzerrungen führen, herausgerechnet werden, ermittelten die Ärzte durch die adjuvante Behandlung eine Verringerung der Wahrscheinlichkeit, im Beobachtungszeitraum zu sterben, um $30 \%$ (Hazard Ratio: 0,70 ; $95 \%$-Konfidenzintervall zwischen 0,64 und 0,76).

In der aktuellen europäischen Leitlinie zum muskelinvasiven Harnblasenkarzinom der EAU (European Association of Urology) von 2015 wird darauf hingewiesen, dass die adjuvante Chemotherapie nach radikaler Zystektomie bei Patienten mit pT3/4 mit oder ohne Lymphknotenbeteiligung, aber ohne klinisch nachweisbare Metastasen noch immer diskutiert wird. Der Vorteil der Adjuvanz liege darin, dass aufgrund des Stagings die Behandlung von Patienten mit niedrigem Risiko für Mikrometastasen vermieden und die Entscheidung für den radikalen Eingriff nicht verzögert werde. Nachteil sei die Gefahr der Übertherapie, weil die Chemosensitivi- tät des Tumors vor Therapiebeginn nicht überprüft werden könne.

Fazit: Nach Angaben unter anderem der Deutschen Krebsgesellschaft liegt die mediane Gesamtüberlebenszeit von $\mathrm{Pa}$ tienten mit einem unbehandelten metastasierten Urothelkarzinom unter sechs Monaten. Eine zusätzliche systemische cisplatinhaltige Polychemotherapie könne die mediane Gesamtüberlebenszeit zwar auf 13 Monate verlängern, allerdings bleibe der palliative Charakter der Chemotherapie bestehen, wie es im Hinweis auf die derzeitige Erarbeitung der deutschen S3-Leitlinie zum Harnblasenkarzinom, die Ende Mai 2016 fertiggestellt sein soll, heißt.

Peter Leiner

Galsky MD et al. Effectiveness of adjuvant chemotherapy for locally advanced bladder cancer. JCO. 2016; doi: 10.1200/JCO.2015.64.1076.

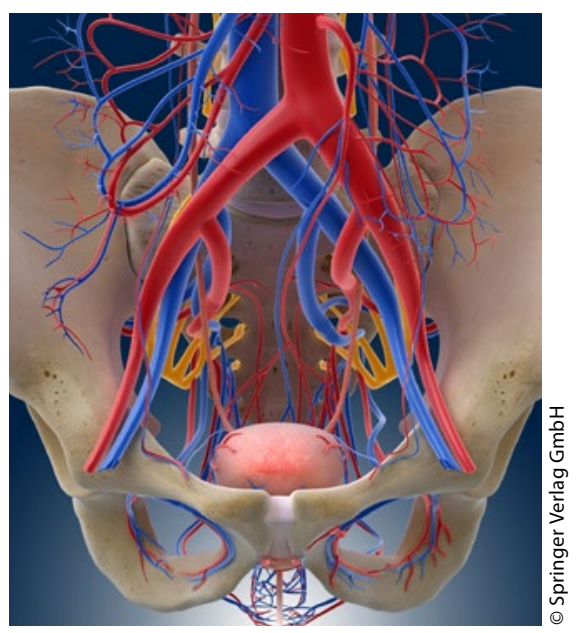

Bessere Aussagen zum Nutzen der adjuvanten Chemo bei Blasenkarzinomen versprach eine kürzlich erschienene Beobachtungsstudie. 